\title{
Comparison of the effects of photon, proton and carbon-ion radiation on the ecto-calreticulin exposure in various tumor cell lines
}

\author{
Yangle Huang $^{1,2}$, Yuanli Dong ${ }^{1,2}$, Jingfang Zhao ${ }^{2,3}$, Lijia Zhang ${ }^{2,3}$, Lin Kong ${ }^{1,2}$, Jiade Jay Lu ${ }^{1,2}$ \\ ${ }^{1}$ Department of Radiation Oncology, Shanghai Proton and Heavy Ion Center, Fudan University Cancer Hospital, Shanghai 201321, China; \\ ${ }^{2}$ Shanghai Engineering Research Center of Proton and Heavy Ion Radiation Therapy, Shanghai 201321, China; ${ }^{3}$ Department of Medical Physics, \\ Shanghai Proton and Heavy Ion Center, Fudan University Cancer Hospital, Shanghai 201321, China \\ Contributions: (I) Conception and design: JJ Lu, L Kong; (II) Administrative support: JJ Lu, L Kong; (III) Provision of study materials or patients: J \\ Zhao, Y Huang, L Zhang; (IV) Collection and assembly of data: Y Huang; (V) Data analysis and interpretation: Y Dong, Y Huang; (VI) Manuscript \\ writing: All authors; (VII) Final approval of manuscript: All authors. \\ Correspondence to: Jiade Jay Lu, MD, MBA; Lin Kong, MD. Shanghai Proton and Heavy Ion Center, 4365 Kangxin Road, Pudong, Shanghai 201321, \\ China. Email: jiade.lu@sphic.org.cn; lin.kong@sphic.org.cn.
}

Background: Accumulating evidence suggested that radiotherapy can activate anti-tumor immune responses by triggering immunogenic cell death (ICD) of tumor cells. Calreticulin is regarded as one of the most important markers of ICD. The cell surface translocation of calreticulin (ecto-CRT) serves as an "eat me" signal for phagocytosis of dying cells, which plays a pivotal role in activating anti-tumor immunity. However, there is limited knowledge describing the effects of proton and carbon-ion radiation on ecto-CRT exposure. Hence, we investigated ecto-CRT exposure in multiple human carcinoma cell lines irradiated by proton and carbon-ion in comparison to photon.

Methods: This study examined four human cancer cell lines including A549 (lung adenocarcinoma), U251MG (glioma), Tca8113 (tongue squamous carcinoma), and CNE-2 (nasopharyngeal carcinoma). Cell lines were irradiated with photon, proton or carbon-ion at 0, 2, 4, $10 \mathrm{~Gy}$ (physical dose). The ecto-CRT exposure level was analyzed by flow cytometry at 12,24 , and $48 \mathrm{~h}$ post-irradiation. The median fluorescence intensity was calculated by FlowJo.

Results: All three types of radial beam increased ecto-CRT exposure of the 4 tumor cell lines in a timedependent manner. Ecto-CRT exposure significantly elevated 1.5-2.4 times over 48 h post-irradiation compared with controls $(\mathrm{P}<0.05)$. Proton and photon increased ecto-CRT exposure with dose escalation. Photon and proton at $10 \mathrm{~Gy}$ increased the most ecto-CRT exposure $(\mathrm{P}<0.05)$, while carbon-ion increased most ecto-CRT exposure at 4 Gy rather than 10 or 2 Gy. When compared with iso-physical dose at $48 \mathrm{~h}$ post-irradiation, proton showed a similar effectiveness with photon. While carbon-ion has significantly stronger effects on increasing ecto-CRT than proton and photon at 2 and 4 Gy, but changed oppositely at 10 Gy $(\mathrm{P}<0.05)$.

Conclusions: All the three types of radiation can increase the ecto-CRT exposure in a time-dependent manner. Proton and photon radiation were equally effective in inducing ecto-CRT exposure, while carbonion revealed a different effectiveness in comparison to photon and proton.

Keywords: Calreticulin; carbon-ion; immunogenic cell death (ICD); proton

Submitted Aug 28, 2019. Accepted for publication Sep 16, 2019.

doi: 10.21037/atm.2019.09.128

View this article at: http://dx.doi.org/10.21037/atm.2019.09.128 


\section{Introduction}

Radiation therapy (RT) plays an important role in treating various malignancies. Whether the intent is palliation or radical cure, radiation used to be considered as a local therapeutic modality through direct killing of tumor cells. However, in recent years, many pre-clinical and clinical researches have revealed the abscopal effect of RT (1). $\mathrm{RT}$ can induce tumor regression in unirradiated regions through indirect anti-tumor immune response (2).

Immunogenic cell death (ICD) of tumor cells is critical in triggering tumor antigen-specific immune response. ICD refers to those cell death forms that can be recognized by the immune system and induce strong anti-tumor immunity (3). Preclinical studies in various human and mouse tumors have demonstrated that RT can induce ICD of tumor cells characterized by the expression of damage associated molecular patterns (DAMPs) (4).

One of the most potent DAMPs released by dying cells after irradiation is the membrane exposure of calreticulin (CRT). CRT is a $\mathrm{Ca}^{2+}$-binding lectin chaperone that is mostly located in the ER lumen (5). When CRT translocated to the surface of dying cells, the ecto-CRT, it can serve as an "eat me" signal for neighboring phagocytes, which plays a pivotal role in activating anti-tumor immunity $(6,7)$. Ecto-calreticulin exposure is strongly correlated with the immunogenicity of tumor cell death induced by chemotherapy as well as RT (8). Thus, elucidating the factors that affect ecto-CRT exposure of tumor cells postirradiation is the key to study the anti-tumor immunity of RT. It is also of great important for further exploration of the combination of radiotherapy and immunotherapy.

Nowadays, ionizing particle radiotherapy, including proton and carbon-ion, is the most advanced and effective RT technology, which has advantages over conventional photon radiation in the treatment of malignancies (9). Ionizing particle can generate stronger radiobiological effects than photon, cause more complicate DNA double strand breaks, resulting in a stronger tumor-killing effects, especially for those photon-resistant tumors $(10,11)$. However, there is limited knowledge of the impacts of different types of radiation beams on immune response. In particular, the differences between ionizing particles and conventional photon beam in inducing ecto-CRT exposure are not well studied yet.

This study aims to investigate the impacts of ionizing particle irradiation on ecto-CRT exposure in comparison to conventional photon irradiation, providing important information for further clinical studies for the combination of ionizing particle radiotherapy and immunotherapy.

\section{Methods}

\section{Cell lines and culture conditions}

The human nasopharyngeal carcinoma cell line, CNE-2 (kindly provided by the Center for Molecular Medicine, Xiangya Hospital, Central South University, Changsha, China), and the human lung adenocarcinoma cell line, A549 (kindly provided by Shanghai Cancer Institute), were cultured in RPMI-1640 supplemented with $10 \%$ fetal bovine serum (FBS). The human tongue squamous carcinoma cell line, Tca8113, and the human glioma cell line, U251 MG (both purchased from Shanghai Zhong Qiao Xin Zhou Biotechnology company), were cultured in DMEM supplemented with $10 \%$ FBS. Cells were grown at $37^{\circ} \mathrm{C}$ with humidified atmosphere and $5 \% \mathrm{CO}_{2}$.

\section{Irradiation}

For photon irradiation, adherent tumor cells in log-growth phase were plated in T25 flasks and irradiated with a $225 \mathrm{kVp}$ $\mathrm{X}$-ray beam (PXi precision X-RAD 225) at a dose rate of $3.198 \mathrm{~Gy} / \mathrm{min}$ as the method previous reported (12). The linear energy transfer (LET) of X-ray was about $2 \mathrm{keV} / \mu \mathrm{m}$.

For proton and carbon-ion irradiation, cells were also plated in T25 flasks (Corning, NY, USA). Then using the IONTRIS intensity-modulated raster scan system with the energy of $173.27 \mathrm{MeV}$, for proton, and $333.82 \mathrm{MeV} / \mathrm{u}$, for carbon-ion, to irradiate the flasks at the Shanghai Proton and Heavy Ion Center (SPHIC), Shanghai, China. The LET was approximately $1.9779 \mathrm{keV} / \mu \mathrm{m}$ for proton and $29.1351 \mathrm{keV} / \mu \mathrm{m}$ for carbon-ion. The beam line was horizontal, and the Bragg Peak of proton and carbon-ion were adjusted to the surface where cell attached with solidwater (see Figure S1).

The irradiation doses, irradiated with photon, proton and carbon-ion, were all physical doses. All the cells, including those mock-irradiated ones, were then washed with fresh medium and incubated at $37{ }^{\circ} \mathrm{C}$ with $5 \% \mathrm{CO}_{2}$.

\section{Flow cytometric analysis of ecto-CRT}

Irradiated and mock-irradiated (control group, 0 Gy) cell lines were detected at 12, 24, and $48 \mathrm{~h}$ after irradiation. Cells were harvested, washed twice with cold PBS. Then 
cells were incubated in cold blocking buffer (5\% FBS in PBS) for 15 min, followed by washing and incubation with monoclonal antibody. Surface exposure of CRT (ecto-CRT) was evaluated by staining with primary labeled monoclonal antibodies (PE-conjugated, specific for human only, Abcam, ab83220). Each sample was then examined by flow cytometer (Guawa easyCyte) to identify ecto-CRT exposure. Flow cytometric data were analyzed by FlowJo software (version 10,FlowJo, LLC, Ashland, Oregon, USA). Forward scatter (FSC) versus side scatter (SSC) plots were gated in order to exclude cellular debris. Median of fluorescence intensity (MFI) was used to compare ectoCRT exposure among different groups.

\section{Statistical analysis}

Statistical analysis was performed using the GraphPad Prism version 7.0 (GraphPad Software, San Diego California, USA). Significant differences among different groups were determined by unpaired Student's $t$-test with a 2 -tailed distribution. $\mathrm{P}$ value $<0.05$ was considered statistically significant.

\section{Results}

All three types of radial beam increase ecto-CRT exposure in a time-dependent manner

First, to compare the time-dependent manner of ectoCRT exposure after irradiated with different radial beams, four human carcinoma cell lines were mock-irradiated (0 Gy) or subjected to photon $10 \mathrm{~Gy}$ (X10Gy), proton 10 Gy (P10Gy) and carbon-ion 4 Gy (C4Gy), respectively. The ecto-CRT exposure level was evaluated at 12, 24 and $48 \mathrm{~h}$ after irradiation by flow cytometry. The fold changes of ecto-CRT exposure levels at 12, 24, and $48 \mathrm{~h}$ postirradiation relative to controls were $0.94-1.12,1.47-1.58$, and 2.07-2.38 respectively for CNE-2 (Figure $1 A$ ), $1.00-1.02,1.07-1.25$, and 1.58-1.68 respectively for A549 (Figure 1B), 1.06-1.12, 1.16-1.29, and 1.45-1.48 respectively for U251 (Figure 1C), 1.00-1.11, 1.00-1.19, and 1.75-1.99 for Tca8113 (Figure 1D). For each cell line and for each type of radial beam, ecto-CRT exposure level increased with time. Ecto-CRT exposure significantly increased over $48 \mathrm{~h}$ post-irradiation with all types of radial beams in all four cell lines compared to controls $(\mathrm{P}<0.05)$ (Figure 1).

\section{Pboton and proton but not carbon-ion increase ecto-CRT exposure in a dose-dependent manner}

Next, to compare the dose-dependent manner of ecto-CRT exposure after irradiated with different irradiation doses, four cell lines were mock-irradiated or treated with 2, 4 and 10 Gy physical dose with photon, proton and carbon-ion, respectively. The ecto-CRT exposure level was evaluated at $48 \mathrm{~h}$ after irradiation by flow cytometry. The fold changes of ecto-CRT exposure levels with X2Gy, X4Gy and X10Gy compared with controls were 1.20-1.32, 1.45-1.65, and 1.95-3.10 for CNE-2, 0.94-1.13, 0.98-1.28, and 1.312.44 for $\mathrm{A} 549,1.03-1.27,1.17-1.42$, and $1.18-1.70$ for U251, 0.97-1.10, 1.12-1.31, and 1.42-2.23 for Tca8113, respectively (Figure 2A). As for P2Gy, P4Gy, and P10Gy, the fold changes of ecto-CRT exposure were 1.01-1.07, 1.79-1.94, and 1.90-2.99 for CNE-2, 0.93-0.97, 1.111.19 , and 1.36-1.89 for A549, 1.14-1.17, 1.32-1.55, and 1.35-1.81 for U251, 1.17-1.25, 1.25-1.35, and 1.22-2.00 for Tca8113, respectively (Figure 2B). For C2Gy, C4Gy, and C10Gy, the fold changes were 1.78-1.84, 1.87-2.21, and 1.41-1.45 for CNE-2, 1.21-1.27, 1.44-1.81, and 1.17-1.37 for A549, 1.21-1.29, 1.26-2.14, and 1.20-1.24 for U251, 1.16-1.32, 1.55-2.24, and 1.08-1.13 for Tca8113, respectively (Figure $2 C$ ). The statistical significances of each group were demonstrated in the bar chat of Figure 2. Both photon and proton increased ecto-CRT exposure with dose escalation. X10Gy and P10Gy increased the most ecto-CRT exposure (Figure 2A,B). However, carbon-ion increased the most ecto-CRT exposure at C4Gy rather than C10Gy or C2Gy (Figure 2C).

\section{Comparison of ecto-CRT exposure irradiated by photon, proton and carbon-ion with iso-physical dose}

Furthermore, we compared the effectiveness of three different radial beams to increase ecto-CRT exposure. Treatment groups were irradiated with 2, 4 and 10 Gy physical dose by photon, proton and carbonion, respectively. The ecto-CRT exposure level was also evaluated at $48 \mathrm{~h}$ post-irradiation by flow cytometry. The significant statistical differences among treatment groups were demonstrated in Figure 3. Proton and photon radiation were equally effective in inducing ecto-CRT exposure when given at iso-physical doses. However, carbon-ion showed a quite different effectiveness with photon. For low dose single irradiation (2 and 4 Gy), carbon-ion can significantly 


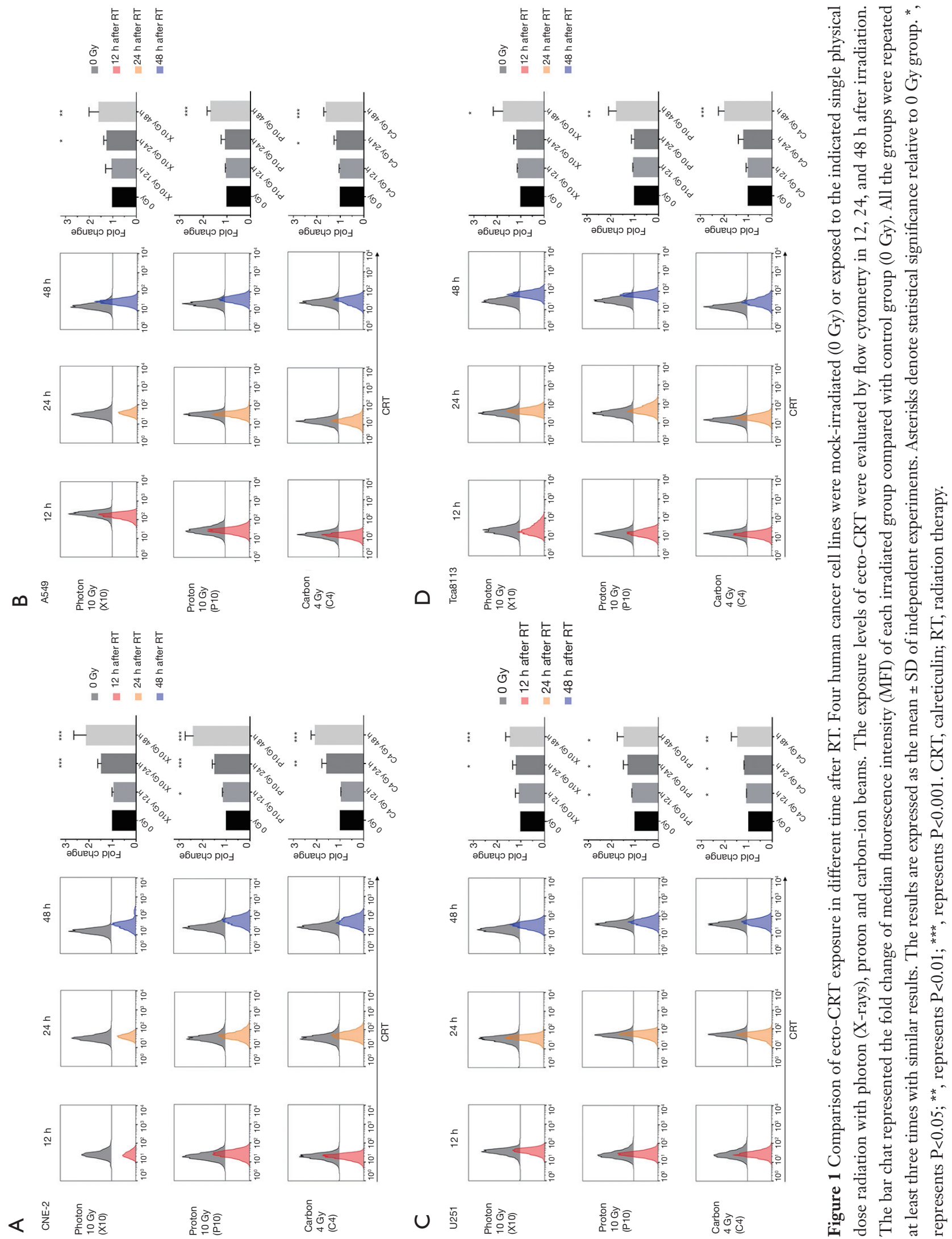


A
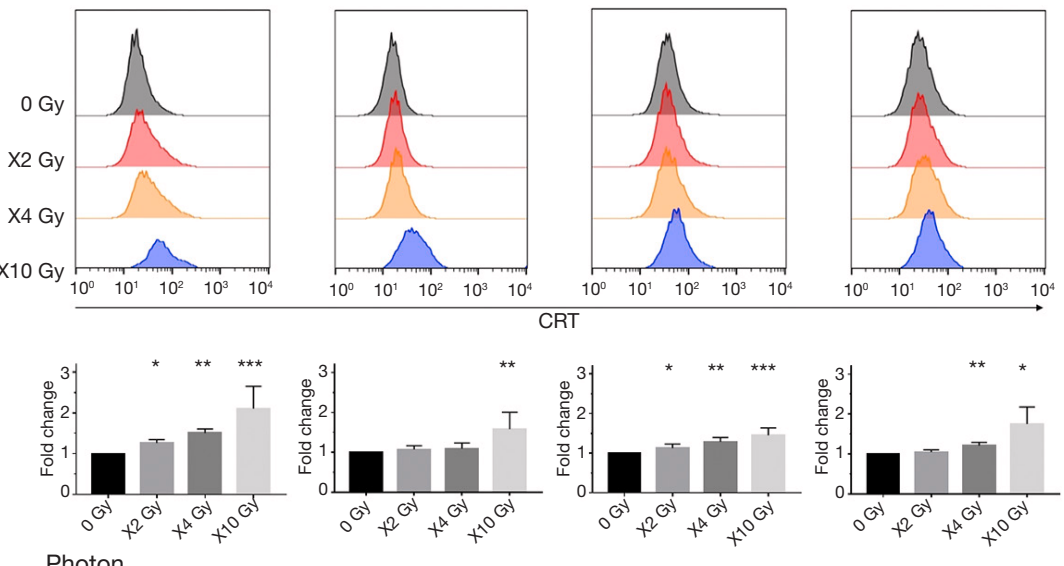

Photon

B

CNE-2
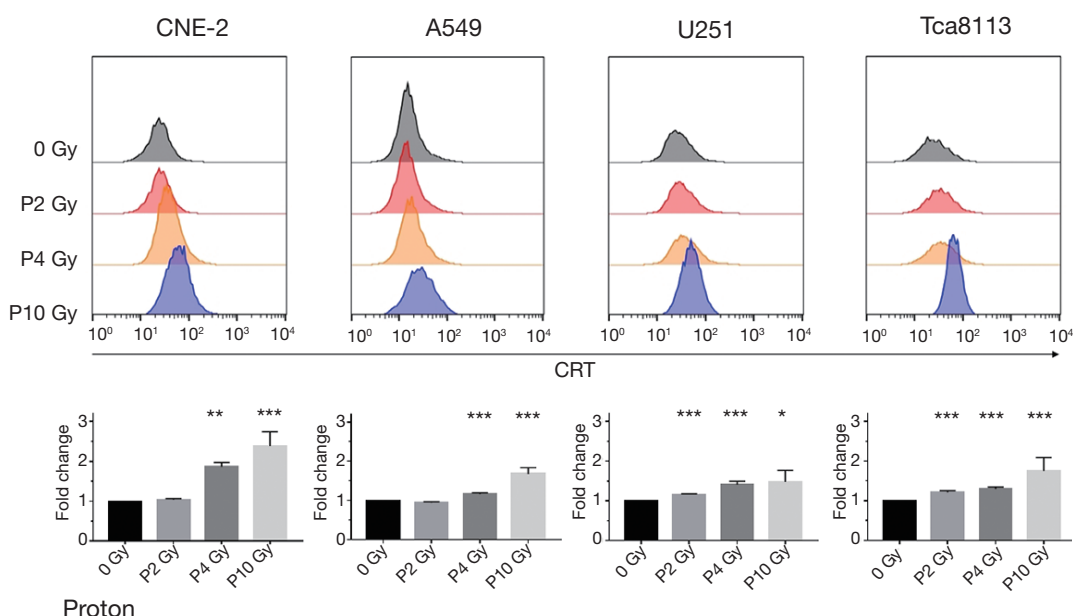

C
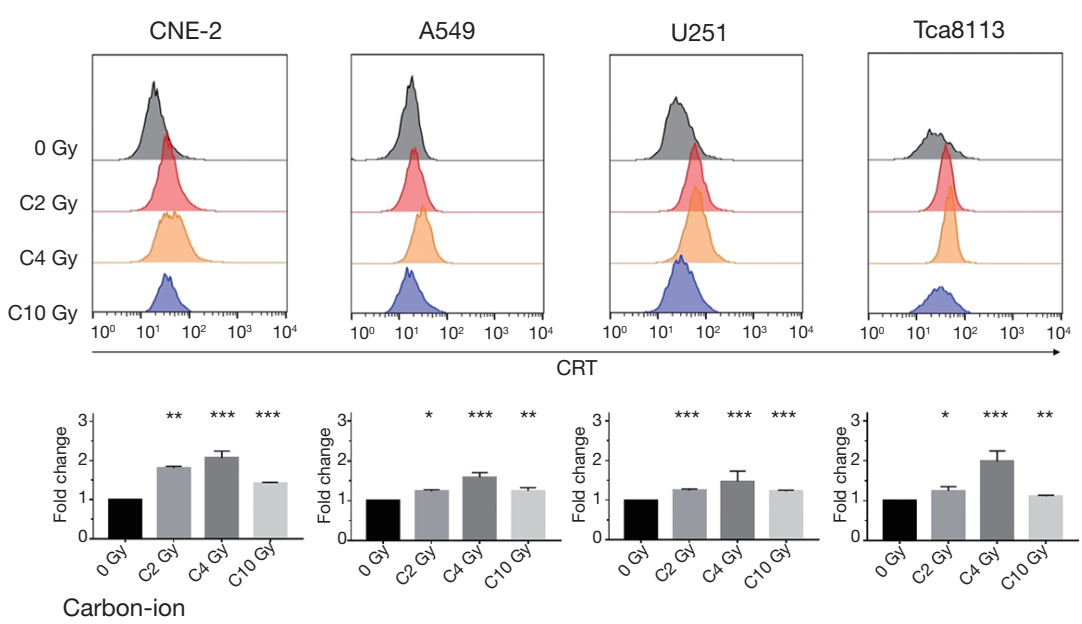

Figure 2 Comparison of ecto-CRT exposure with different physical doses. Four human cancer cell lines were mock-irradiated (0 Gy) or exposed to 2, 4 and 10 Gy with photon, proton and carbon-ion beams, respectively. The exposure levels of ecto-CRT were evaluated by flow cytometry at $48 \mathrm{~h}$ after irradiation. The bar chat represented the fold change of median fluorescence intensity (MFI) of each irradiated group compared with control group ( 0 Gy). All the groups were repeated at least three times with similar results. The results are expressed as the mean $\pm \mathrm{SD}$ of independent experiments. ${ }^{*}$, represents $\mathrm{P}<0.05$; $^{* *}$, represents $\mathrm{P}<0.01$; ${ }^{* * *}$, represents $\mathrm{P}<0.001$. CRT, calreticulin. 

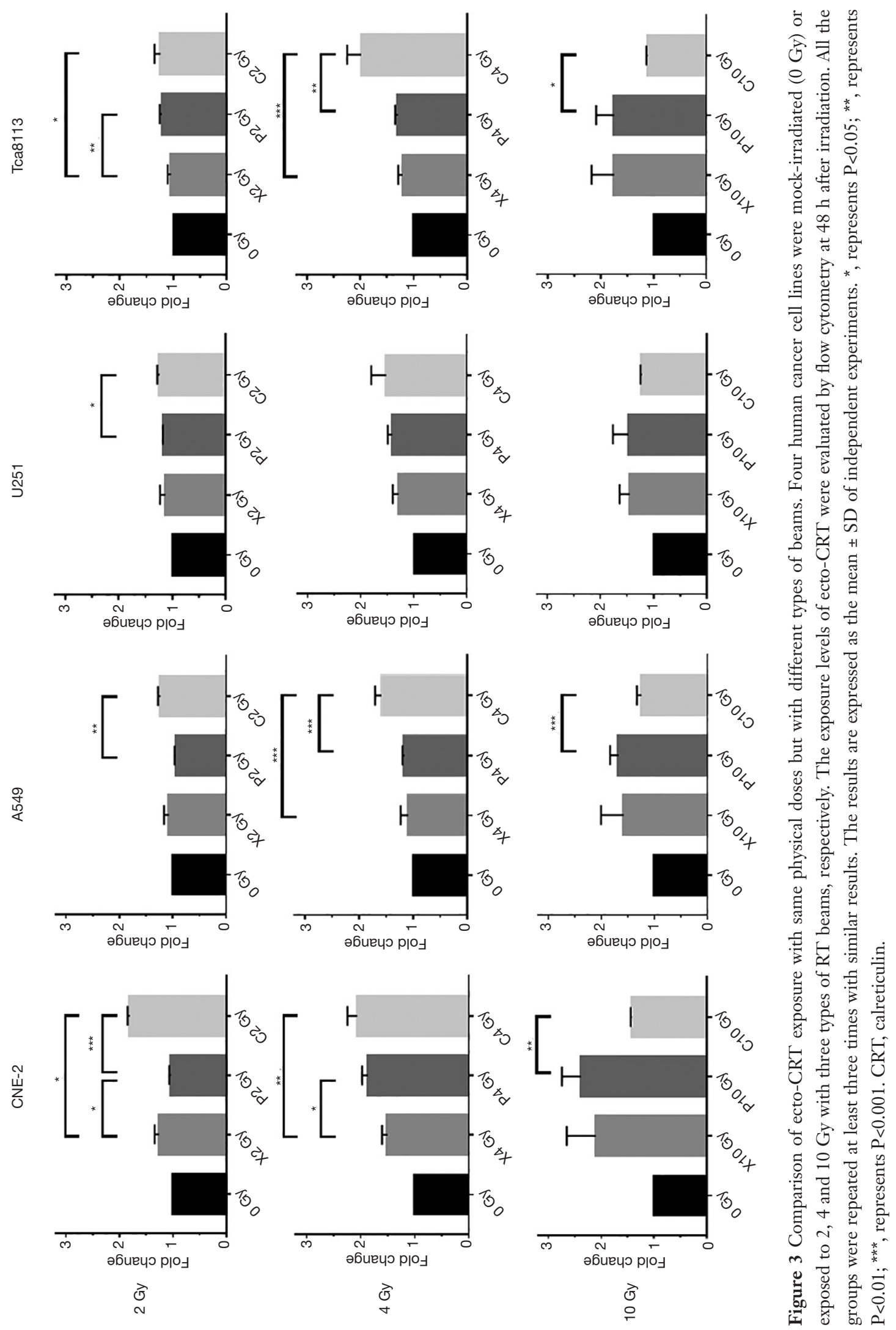
improve the exposure level of ecto-CRT than photon in CNE-2, A549 and Tca8113. Although the results showed no statistically significant differences in U251, there was a trend that carbon-ion could increase more ecto-CRT exposure than photon. However, for relatively high dose single irradiation (10 Gy), photon can reversely increase more ecto-CRT exposure than carbon-ion, although the differences were not statistically significant. But, when compared proton with carbon-ion at $10 \mathrm{~Gy}$, proton can significantly increase the ecto-CRT exposure than carbonion in various cell lines except for $\mathrm{U} 251(\mathrm{P}<0.05)$ (Figure 3).

Taken together, all the three types of radial beam increased ecto-CRT exposure of various human cancer cell lines in a time-dependent manner. Photon and proton increased ecto-CRT exposure in a dose-dependent manner, but carbon-ion did not. Proton revealed a similar effectiveness with photon to induce ecto-CRT exposure, while carbon-ion showed a quite different effectiveness in comparison to photon and proton.

\section{Discussion}

Nowadays, a growing body of research have reported the abscopal effect and the anti-tumor immune response contributed by RT (13). Induction of immunogenic cancer cell death has become one of the major aims of RT (14), because it would be of benefit not only to treat distant and occult metastasis but also to eradicate radiationresistant cancer cells and cancer stem cells. As the frontier technology of RT, proton and carbon-ion therapy may provide a promising future to conquer cancer. However, the effectiveness of proton and carbon-ion to trigger immune response against dying tumor cells remain unclear. This present study evaluated the level of ecto-CRT exposure in four human cancer cell lines treated with photon, proton and carbon-ion, providing the reference for future research related to ICD induced by RT, especially for proton and carbon-ion irradiation.

As mentioned before, CRT plays an important role in ICD and inducing effective anti-tumor immune response. The ecto-CRT functioned as an "eat-me" signal, promoting the phagocytosis of dying tumor cells by DCs (6). Blockade of ecto-CRT or knockdown of CRT will annihilate the immunogenicity of cell death $(8,15)$. Furthermore, for clinical study, some researches have reported the diagnostic and prognostic value of CRT in various tumor (16-19). It was found that high CRT expression on tumor cells was associated with a higher density of infiltrating mature DC and effective $T$ cells, accordingly with a better prognosis in lung cancer patients (20). However, the ecto-CRT exposure level in patients after treated with RT, especially proton and carbon-ion, was lack of study, because of the difficulty to obtain tumor samples post-RT. Thus, we conducted this in vitro experiment, trying to figure out the post-irradiated ecto-CRT exposure in tumor cells treated with RT, especially with proton and carbon-ion.

Contrasting with the kinetics of ecto-CRT exposure induced by chemotherapy, like anthracycline and oxaliplatin, which elicited CRT exposure in minutes after treatment $(3,21)$, we found that all the three types of irradiation (photon, proton and carbon-ion) increased ecto-CRT exposure over time. The ecto-CRT exposure significantly increased at $48 \mathrm{~h}$ after irradiation, while at $12 \mathrm{~h}$ post-irradiation, there were just slightly increase of the ecto-CRT in various tumor cell lines (Figure 1). This finding was consistent with previous study, which was about photon radiation-induced ICD parameter in human HNSCC cell lines (22). They evaluated the membrane CRT exposure at $15 \mathrm{~h}$ after photon radiation, and the results showed no significant increase in CRT exposure. Another research observed a time-dependent manner of ecto-CRT increase induced by photon irradiation in breast carcinoma cell (23). All these results suggested that RT induced ectoCRT exposure in a time-dependent manner, which is at variance with chemotherapy.

When comparison of ecto-CRT exposure with different doses of each radical beam, we found that photon and proton induced ecto-CRT exposure with dose increasing. However, carbon-ion exhibited a different manner, it increased most ecto-CRT exposure at C4Gy rather than C10Gy or C2Gy (Figure 2). These results indicated that each radical beam has an optimal dose to induce ectoCRT as well as ICD of tumor cells. It has already been studied in photon irradiation that only using a proper range of fractionation dose (7-15 Gy), RT can be immunestimulatory, otherwise it may induce no immune response or even immune-suppressive effects (24-27). As we compared with carbon-ion, proton and photon irradiation, we must take the relative biological effectiveness (RBE) into consideration. The RBE of proton is considered as 1.1 (28). So proton usually showed a similar biological effect with photon, so did in our research. Previous research also demonstrated that proton shared a common immunogenic modulation with photon (29). However, carbon-ion was thought to have favorable biological property with a higher RBE value as $2-3(30,31)$. Our 
previous study suggested that the RBE of carbon-ion is 2.5 for CNE-2 cell line (12). So carbon-ion 2 and 4 Gy physical dose are equivalent to 4-6 and 8-12 GyE equivalent biological dose. While carbon-ion 10 Gy physical dose might be equivalent to 20-30 GyE equivalent biological dose. Thus, when compared three types of radical beams in iso-physical dose, we found that carbon-ion has stronger effects on increasing ecto-CRT than proton and photon at low dose (2, 4 Gy), but changed oppositely at high dose (10 Gy) (Figure 3). We assumed that C10Gy physical dose is relatively too high for inducing ICD of tumor cells, so cells exposed to such high dose radiation may go through a different death pattern. Further study is warranted to elucidate the mechanism underlying this phenomenon.

In this study, we included four human carcinoma cancer cell lines. When compared the fold change of ecto-CRT irradiated with iso-physical dose (Figures 1 and 2), we found that the increase of ecto-CRT is tumordependent. RT with each type radical beam can increase more ecto-CRT exposure in radiation-sensitive tumor cell, such as nasopharyngeal carcinoma (CNE-2) and tongue squamous carcinoma (Tca-8113), than radiation-resistant cell, like glioblastoma (U251) and lung adenocarcinoma (A549). It suggested that tumor cells may have different immunogenicity when treated with RT. In addition, the RBE values of different tumor are quite different. As we previously reported, the RBE of carbon-ion in CNE-2 cell line was 2.5 (12). While for U251 cell line, the RBE of carbon-ion was only 1.43 (32). Thus, it was reflected in our results that carbon-ion did not significantly increase more ecto-CRT exposure than proton and photon for U251 cell line when compared with iso-physical dose (Figure 3).

In conclusion, proton and carbon-ion as well as photon radiation can increase the ecto-CRT exposure in a timedependent manner. Proton elicited ecto-CRT exposure in a similar pattern with photon, while carbon-ion revealed a different effectiveness on ecto-CRT exposure at variance with photon and proton radiation. Our results offered a preliminary result about the immunogenic effects of proton and carbon-ion in comparison with photon, though further detailed research is needed to provide more rational information and solid evidence for the use of RT, especially proton and carbon-ion, in combination with immunotherapy.

\section{Acknowledgments}

We deeply appreciate BS Dwarakanath and Yun Sun
(Division of Research and Development, Shanghai Proton and Heavy Ion Center, Shanghai 201321, China) for their kind help.

Funding: This work was supported by National Key Research and Development Program of China (Project No. 2017YFC0108603), Science and Technology Commission of Shanghai Municipality (Project No. 19XD1432900), Shanghai Municipal Health Commission (Project No. 201640024), and Science and Technology Development Fund of Shanghai Pudong New Area (Project No. PKJ2016-Y41).

\section{Footnote}

Conflicts of Interest: The authors have no conflicts of interest to declare.

Etbical Statement: The authors are accountable for all aspects of the work in ensuring that questions related to the accuracy or integrity of any part of the work are appropriately investigated and resolved.

\section{References}

1. Reynders K, Illidge T, Siva S, et al. The abscopal effect of local radiotherapy: using immunotherapy to make a rare event clinically relevant. Cancer Treat Rev 2015;41:503-10.

2. Demaria S, Formenti S. Radiotherapy Effects on AntiTumor Immunity: Implications for Cancer Treatment. Front Oncol 2013;3:128.

3. Obeid M, Panaretakis T, Tesniere A, et al. Leveraging the immune system during chemotherapy: Moving calreticulin to the cell surface converts apoptotic death from "silent" to immunogenic. Cancer Res 2007;67:7941-4.

4. Garg AD, Dudek-Peric A, Romano E, et al. Immunogenic cell death. Int J Dev Biol 2015;59:131-40.

5. Gelebart P, Opas M, Michalak M. Calreticulin, a Ca2+binding chaperone of the endoplasmic reticulum. Int $\mathrm{J}$ Biochem Cell Biol 2005;37:260-6.

6. Gardai SJ, McPhillips K, Frasch S, et al. Cell-surface calreticulin initiates clearance of viable or apoptotic cells through trans-activation of LRP on the phagocyte. Cell 2005;123:321-34.

7. Suzuki Y, Mimura K, Yoshimoto Y, et al. Immunogenic tumor cell death induced by chemoradiotherapy in patients with esophageal squamous cell carcinoma. Cancer Res 2012;72:3967-76. 
8. Panaretakis T, Kepp O, Brockmeier U, et al. Mechanisms of pre-apoptotic calreticulin exposure in immunogenic cell death. EMBO J 2009;28:578-90.

9. Jiang GL. Particle therapy for cancers: a new weapon in radiation therapy. Front Med 2012;6:165-72.

10. Durante M, Orecchia R, Loeffler J. Charged-particle therapy in cancer: clinical uses and future perspectives. Nat Rev Clin Oncol 2017;14:483-95.

11. Kamada T, Tsujii H, Blakely E, et al. Carbon ion radiotherapy in Japan: an assessment of 20 years of clinical experience. Lancet Oncol 2015;16:e93-100.

12. Bao C, Sun Y, Dong Y, et al. The relative biological effectiveness of proton and carbon ion beams in photonsensitive and resistant nasopharyngeal cancer cells. Transl Cancer Res 2018;7:170-9.

13. Frey B, Rückert M, Deloch L, et al. Immunomodulation by ionizing radiation-impact for design of radioimmunotherapies and for treatment of inflammatory diseases. Immunol Rev 2017;280:231-48.

14. Golden EB, Apetoh L. Radiotherapy and Immunogenic Cell Death. Semin Radiat Oncol 2015;25:11-7.

15. Garg AD, Krysko D, Verfaillie T, et al. A novel pathway combining calreticulin exposure and ATP secretion in immunogenic cancer cell death. EMBO J 2012;31:1062-79.

16. Liu R, Gong J, Chen J, et al. Calreticulin as a potential diagnostic biomarker for lung cancer. Cancer Immunol Immunother 2012;61:855-64.

17. Chiang WF, Hwang T, Hour T, et al. Calreticulin, an endoplasmic reticulum-resident protein, is highly expressed and essential for cell proliferation and migration in oral squamous cell carcinoma. Oral Oncol 2013;49:534-41.

18. Hsu WM, Hsieh F, Jeng Y, et al. Calreticulin expression in neuroblastoma--a novel independent prognostic factor. Ann Oncol 2005;16:314-21.

19. Wemeau M, Kepp O, Tesniere A, et al. Calreticulin exposure on malignant blasts predicts a cellular anticancer immune response in patients with acute myeloid leukemia. Cell Death Dis 2010;1:e104.

20. Fucikova J, Becht E, Iribarren K, et al. Calreticulin expression in human non-small cell lung cancers correlates with increased accumulation of antitumor immune cells and favorable prognosis. Cancer Res 2016;76:1746-56.

21. Tesniere A, Schlemmer F, Boige V, et al. Immunogenic death of colon cancer cells treated with oxaliplatin. Oncogene 2010;29:482-91.
22. Schneider K, Bol V, Grégoire V. Lack of differences in radiation-induced immunogenicity parameters between HPV-positive and HPV-negative human HNSCC cell lines. Radiother Oncol 2017;124:411-7.

23. Gameiro SR, Jammeh M, Wattenberg M, et al. Radiationinduced immunogenic modulation of tumor enhances antigen processing and calreticulin exposure, resulting in enhanced T-cell killing. Oncotarget 2014;5:403-16.

24. Brooks ED, Schoenhals JE, Tang C, et al. Stereotactic Ablative Radiation Therapy Combined With Immunotherapy for Solid Tumors. Cancer J 2016;22:257-66.

25. Chang JY, Bezjak A, Mornex F. Stereotactic ablative radiotherapy for centrally located early stage non-smallcell lung cancer: what we have learned. J Thorac Oncol 2015;10:577-85.

26. Nishikawa H, Sakaguchi S. Regulatory T cells in tumor immunity. Int J Cancer 2010;127:759-67.

27. Bernstein MB, Krishnan S, Hodge J, et al. Immunotherapy and stereotactic ablative radiotherapy (ISABR): a curative approach? Nat Rev Clin Oncol 2016;13:516-24.

28. Schulz-Ertner D, Tsujii H. Particle radiation therapy using proton and heavier ion beams. J Clin Oncol 2007;25:953-64.

29. Gameiro SR, Malamas A, Bernstein M, et al. Tumor Cells Surviving Exposure to Proton or Photon Radiation Share a Common Immunogenic Modulation Signature, Rendering Them More Sensitive to T Cell-Mediated Killing. Int J Radiat Oncol Biol Phys 2016;95:120-30.

30. Held KD, Kawamura H, Kaminuma T, et al. Effects of Charged Particles on Human Tumor Cells. Front Oncol 2016;6:23.

31. Kong L, Gao J, Hu J, et al. Carbon ion radiotherapy boost in the treatment of glioblastoma: a randomized phase I/III clinical trial. Cancer Commun (Lond) 2019;39:5 .

32. Ferrandon S, Magné N, Battiston-Montagne P, et al. Cellular and molecular portrait of eleven human glioblastoma cell lines under photon and carbon ion irradiation. Cancer Lett 2015;360:10-6.

Cite this article as: Huang Y, Dong Y, Zhao J, Zhang L, Kong L, Lu JJ. Comparison of the effects of photon, proton and carbon-ion radiation on the ecto-calreticulin exposure in various tumor cell lines. Ann Transl Med 2019;7(20):542. doi: 10.21037/atm.2019.09.128 


\section{Supplementary}
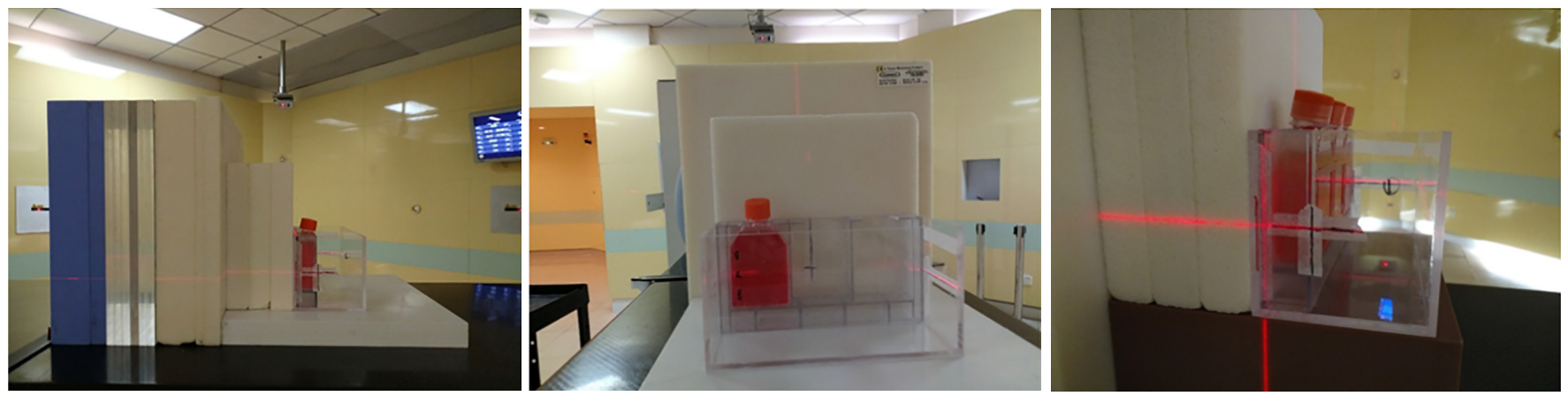

Figure S1 The irradiation mold of proton and carbon-ion irradiation. The beam lines of proton and carbon-ion were all horizontal. The Bragg Peak of proton and carbon-ion were adjusted to the surface where cell attached with solid-water. 